\title{
Reflections on the Discovery of Pulsars
}

\section{Jocelyn Bell Burnell}

University of Oxford

Denys Wilkinson Building, Keble Rd, Oxford OX1 3RH, UK

jocelyn@astro.ox.ac.uk

In this short paper I will give a brief account of the discovery of pulsars, report several other 'near misses' when pulsars were almost discovered and finally reflect on the factors that led to the discovery and what lessons we might learn.

Accelerating the Rate of Astronomical Discovery - sps5

Rio de Janeiro, Brazil

August 11-14 2009 


\section{Introduction}

In summer 1965 Antony Hewish of the Cambridge Radio Astronomy group was awarded a grant of $£ 10000$ to build a radio telescope that would use interplanetary scintillation to detect and measure the angular diameters of quasars. Margaret Clarke had been the first to notice that radio sources which were believed to be compact showed scintillation (fluctuations in brightness) whereas more extended sources did not. The cause of the scintillation was electron density fluctuations in the solar wind, and the timescale was sub-second. As the solar wind expanded away from the sun the angular diameter of these clouds of enhanced electron density increased. So the scintillation of a source changed during the year as the line of sight to it passed the sun at different distances and through clouds of different angular extent.

I joined the radio astronomy group that summer as Tony's newest $\mathrm{PhD}$ student, to work on this project. The first two years were spent building the radio telescope - $\mathrm{a}$ 1.8 hectare array of 2048 dipoles, operating at a frequency of $81.5 \mathrm{MHz}$. A short time constant $(0.1 \mathrm{~s})$ was to be used, so as not to smear out the scintillation, and to give reasonable signal-to-noise the large area antenna was required. The telescope was an interferometer operating as a transit instrument which, by inserting phase delays, could be steered in declination.

Four beams, at different declinations, were observed at any one time, with the output going onto four pen-recorders (no computing facilities were available). The declination range $-8^{\circ}<\delta<44^{\circ}$ was repeatedly mapped to identify quasars and to measure their angular diameter. I was responsible for the first six months' observing and the data analysis.

\section{The discovery of pulsars}

The pen recorders produced $32 \mathrm{~m}$ of paper chart per day. One complete sky scan generated $128 \mathrm{~m}$ and in the six months I ran the survey I acquired $5.8 \mathrm{~km}$ of paper chart in total. The pen traces showed scintillating quasars and random bursts of artificial interference. Occasionally, in the middle of the night (when one would not expect much interplanetary scintillation), $0.5 \mathrm{~cm}$ in the $128 \mathrm{~m}$ showed an unusual signal.

After a month of special observations and null results the source showed itself to be a string of pulses, with a period of about $1.33 \mathrm{~s}$. The next month was spent checking the equipment and the signal, and continued observation of the source. Was it local radio interference? No-it kept sidereal time. Was it a fault with the equipment? Nothe signal was picked up by a separate telescope and receiver. It was small (as implied by the fast pulse period) and it was big (it maintained the pulse period accurately). We could not initially get our heads round that one! Dispersion measurement showed it was 200 light years away (i.e. beyond the Solar System, but nearby in the Milky Way) Was it Little Green Men- extraterrestrials? If so, they'd be on a planet orbiting their Sun. No - there was no Doppler effect (other than that due to the motion of the Earth round the Sun). 
Then, late one evening doing some routine chart analysis, I noticed what seemed to be another source with similarly unusual signal, and observations in the cold early hours of the morning confirmed a second source, this time with a period of about $1.25 \mathrm{~s}$. This strongly suggested that I had stumbled on a new kind of stellar population. I found pulsars numbers three and four a few weeks later.

\section{Crab pulsar almost discovered}

Some other astronomers have kindly related to me how they 'nearly discovered pulsars' and I repeat some of the stories here. I concentrate on the stories where pulses were actually seen, rather than on the 'if only' kind of story.

The first dates from summer 1957 when Elliott Moore helped out at a public observing night at the 82 inch McDonald Observatory telescope (now known as the Otto Struve telescope). For the final observation of the night the telescope was set on the inner part of the Crab Nebula. One member of the public was a young woman probably in her late twenties; she was familiar with the night sky because her job was flying planes and in the dark cockpit there was little else to do except watch the stars. After her turn at the telescope she reported that one of the stars in the Crab nebula was blinking rapidly, and described which one. Elliott Moore looked but could not see the effect. He suggested it was scintillation, but later realised that with such a large aperture telescope it couldn't be.

The second dates from December 1965. Sue Simkin was taking UV spectra with the Carnegie Image Spectrograph at the Kitt Peak 84 inch telescope. She was asked by Lo Woltjer to take a UV spectrum of Minkowski's star (now known to be the pulsar). She said the spectrum was dull, but as she took it she observed flickering, or as if there were waves going out from it. Lo maintained there couldn't be, but after the discovery of the pulsar agreed that must have been what she had seen. [The Crab pulsar, with a period of $30 \mathrm{~Hz}$, is sufficiently fast that many people cannot see it; however some people can see $30 \mathrm{~Hz}$ and Sue Simkin knows she is one of them - as a child she lived in an area where the mains power supply was at $30 \mathrm{~Hz}$.]

The final example of possible detection of the Crab pulsar concerns Charles Schisler, a USAF technician, stationed in 1967-8 at a Ballistic Missile Early Warning Site in Alaska. Using the radars he found fourteen sources, ten or eleven of which have been identified to be pulsars. I am not clear that he detected pulses, but he certainly identified variable sources. The first he detected was the Crab pulsar. His Air Force superiors did not appreciate the significance of his observation and he was not able to publish his results.

\section{PSR0329+54 almost discovered}

In Europe in the mid-nineteen sixties the northern sky was being sensitively surveyed at $408 \mathrm{MHz}$. During what the observers hoped was the final week of observing, one of the pen recorders was misbehaving, and in the early hours of one morning the pen in that recorder started rhythmically sweeping across the chart paper. The observer on duty cursed, and thumped the pen recorder, whereupon the pen returned to its normal behaviour. The observer said 'Good!', put on his coat and went home! He had made the first observation of PSR 0329+54; it had passed out of the 
telescope beam at the point he thumped the pen recorder! Unfortunately he did not make an entry in the log book, and nobody searched the data following the discovery of the first pulsars (which did not include 0329+54).

\section{Key factors and reflections}

The following issues seem to be significant:

I had my own telescope and receivers, and I understood their behaviour - I was not using 'common user' facilities; I scrutinised all the data and as a research student I had time/space to follow-up anomalies; I knew less astrophysics than my supervisor, so did not see why this signal had to be artificial interference - a little ignorance, or the ability to ignore the conventional wisdom, is advantageous. If we had computerised the search, would the pulsars have been discovered - would we have programmed the computer in a sufficiently open way to pick up such an unexpected signal?

The discovery resulted from careful work - sharp observation and conscientious follow-up. Pursuit of anomalies and follow up requires time; we were not aware of other observers likely to make and announce the discovery before us and so we were not tempted to short-cut the follow-up. We also had funding, at least for the immediate future.

This was not one of the objectives, or targets of the programme - by definition it could not have been.

This was one of the first observations with a short time constant (i.e. we had moved into a new area of phase space)

We had a good address; an outrageous discovery made by a reputable laboratory is more likely to get published (quickly).

How difficult it is to predict how and where breakthroughs will come; serendipitous ones of course are by definition even harder to predict. Although the facilities I used were not particularly good, the Cambridge ambiance and the exciting atmosphere that then pertained in radio astronomy probably helped contemplation of the incredible. 